\title{
Development of a displacement measurement system for Wendelstein 7-X superconducting magnet system
}

\author{
P. Chen, C. J. Caldwell-Nichols, J. P. Kallmeyer, M. Nitz and F. Scherwenke
}

\begin{abstract}
The superconducting magnet system of the Wendelstein 7-X (W7-X) stellarator fusion experiment consists of 50 non-planar and 20 planar coils. During operation, the W7-X stellarator will produce a magnetic field strength of 3 $T$ in the plasma centre, and it will also bring very high electromagnetic forces in the coils and their related mechanical support structures. In order ensure the mechanical integrity of the superconducting magnet system, 70 devices of a displacement measurement system are being installed to monitor the coil displacement. In this paper, the development of the displacement measurement system and the first measurement results performed on a W7-X superconducting planar coil are presented.
\end{abstract}

Index Terms-Stellarator, Wendelstein 7-X, Superconducting coil, Displacement measurement

\section{INTRODUCTION}

HE Wendelstein 7-X (W7-X) stellarator fusion experiment is presently under construction

$T_{\text {at }}$ the Max-Planck-Institut fuer Plasmaphysik (IPP) in Greifswald, Germany [1]. The W7-X stellarator uses 50 non-planar and 20 planar superconducting coils to produce a magnetic field strength of $3 \mathrm{~T}$ in the plasma centre. The coils will be fixed by the central support structure and inter-coil structural elements. The coils are arranged in a toroidal configuration around the plasma vessel. The coils are divided into five equal modules (five-fold symmetry), each of which consists of two symmetric half modules [2]. In each half module, five differently shaped non-planar coils and two slightly different planar coils (see Fig.1) are bolted to a massive steel structure.

In order to monitor the integrity of the superconducting magnet system and its related components during normal and abnormal operation of W7-X, a displacement measurement system was included in the machine design. There will be a total of 70 devices in the displacement measurement system installed around the whole machine, which will be placed in key positions defined by finite element calculations.

Due to the extreme operational environment (vacuum, high

P. Chen is now with the Key Laboratory of Applied Superconductivity, Institute of Electrical Engineering, Chinese Academy of Sciences, Beijing 100080, China and the Graduate University of Chinese Academy of Sciences, Beijing 100039, China (e-mail: chenpeng@mail.iee.ac.cn).

C. J. Caldwell-Nichlos, J. P. Kallmeyer, M. Nitz and F. Scherwenke are with the Max-Plack-Institut für Plasmaphysik, D-17489 Greifswald, Germany. 


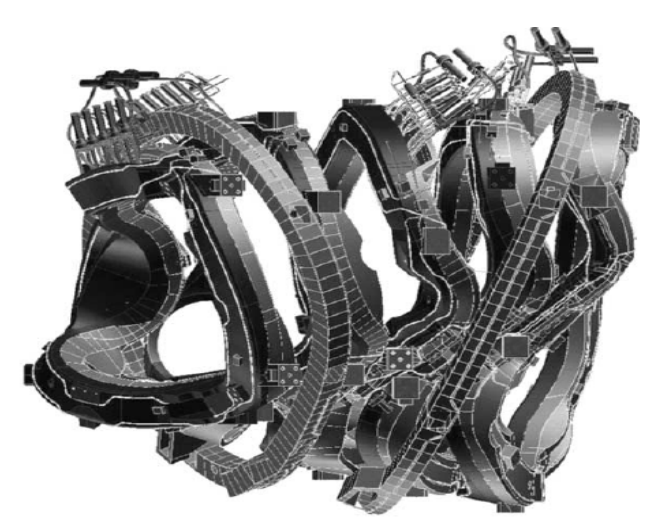

Fig. 1. Half module of the W7-X superconducting coil system.

magnetic field and low temperature), the accurate measurement of displacement is a challenge. A commercial cantilever based displacement measurement system was designed and tested in the laboratory. In addition, during the acceptance test process of the planar coil AAC33 at the Commissariat a l'Energie Atomique (CEA) in Saclay, France, two measurement systems were applied and tested.

In this paper, the design and investigations of the displacement measurement system and the first measurements performed on a W7-X superconducting planar coil are presented.

\section{SENSOR DESIGN AND TEST}

A. Sensor Design

The displacement measurement system is based on the commercial strain type displacement transducer CE-10SL, which is manufactured by Tokyo Sokki Kenkyuio Co., Ltd. The configuration of the transducer is shown in Fig.2.

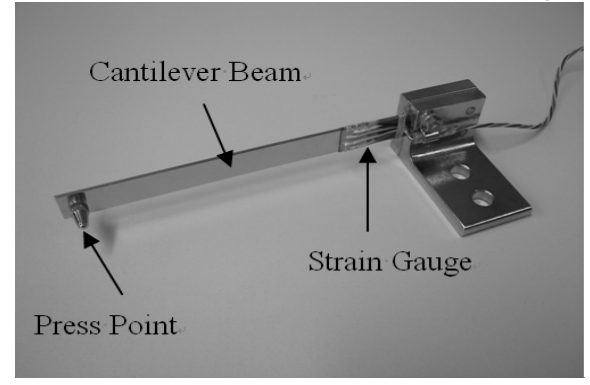

Fig. 2. The commercial strain type displacement transducer.

The displacement transducer is in the form of a cantilever. The cantilever beam is made of the antimagnetic material beryllium copper, its weight (including mounting plate) is $95 \mathrm{~g}$, and the resonance frequency is $25 \mathrm{~Hz}$ [3]. The displacement measurement capacity of this transducer is $\pm 10 \mathrm{~mm}$, the resolution is better than $5 \mu \mathrm{m}$ and the nonlinearity is $0.4 \%$. The maximum exciting voltage is $10 \mathrm{~V}$ and the maximum force on the contact point ("press point" in Fig.2) is $3.2 \mathrm{~N}$. This cantilever transducer can operate well in the environment of $4 \mathrm{~K}$ and maximum $5.5 \mathrm{~T}$ magnetic fields.

There are four low temperature strain gauges mounted on the cantilever beam, and each 
gauge resistance is $350 \Omega$. The four strain gauges make up a Wheatstone full bridge circuit, which is shown in Fig.3.

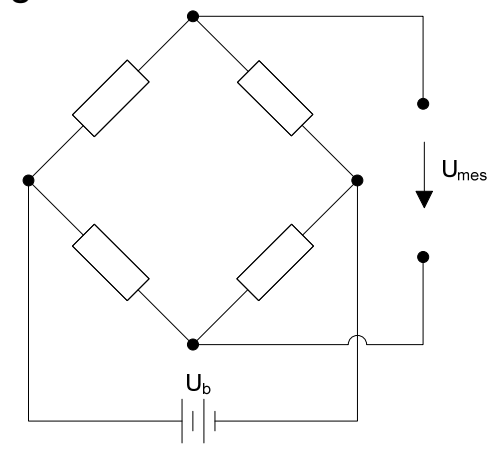

Fig. 3. Wheatstone full bridge circuit.

The configuration of the measurement system is shown in Fig.4. It consists of a cantilever beam, aluminum support structure, pull spring (Inconel X-750), copper wire $(\varnothing 0.4 \mathrm{~mm})$ and solder terminals. The support structure will be installed on the surface of the measurement object. The cantilever beam is fixed on the support structure by four M3 screws. All the electrical circuitry can be accessed through the connection at the solder terminals. At first, with the defined spring pull force, the cantilever beam is displaced in the negative direction. Then, the beam is pulled back to the zero position by the copper wire. The other end of the copper wire is fixed to the second measurement object. In this way, the displacement measurement system can measure the relative displacement between the two objects both in negative and positive directions $(-10 \mathrm{~mm}$ to $+10 \mathrm{~mm})$.

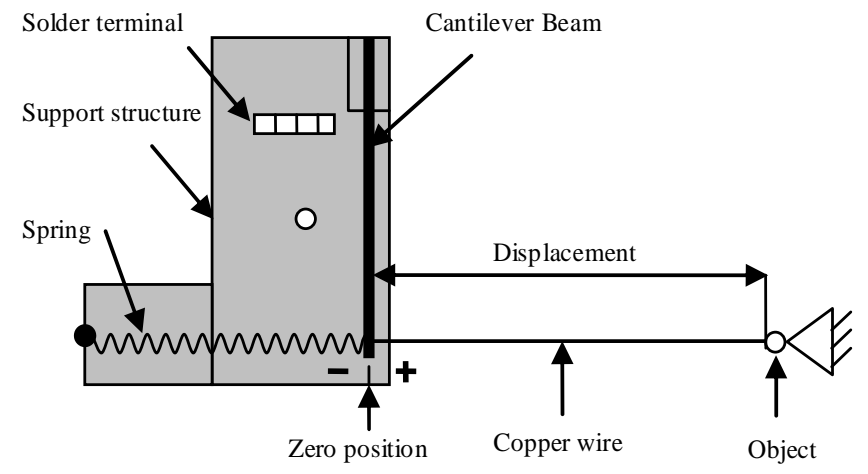

Fig. 4. The schematic of the displacement measurement system.

The simplified mechanical model of the displacement measurement system consists of three springs, which is shown in Fig.5. $\mathrm{K}_{1}$ is the spring constant of the copper wire. If the Young's modulus (E), cross section (S) and length (L) of the wire are given, $K_{1}$ can be calculated by $\mathrm{K}_{1}=(\mathrm{E} \times \mathrm{S}) / \mathrm{L} . \mathrm{K}_{2}$ is the spring constant of the cantilever beam, which is $0.32 \mathrm{~N} / \mathrm{mm}$. $K_{3}$ is the spring constant of the Inconel spring, which is $0.1 \mathrm{~N} / \mathrm{mm}$. $D_{B}$ is the displacement of the joint among cantilever beam, pull spring and wire (Point $B$ ), which is also the displacement measured by the cantilever. According to the definition of commercial cantilever sensitivity, $D_{B}(m m)$ can be calculated by $D_{B}=U_{\text {mes }} /\left(K_{0} \times U_{b}\right)$, where 
$\mathrm{K}_{0}$ is the sensor sensitivity $(\mathrm{mV} / \mathrm{V} / \mathrm{mm}), \mathrm{U}_{\mathrm{b}}$ is the exciting power $(\mathrm{V}), \mathrm{U}_{\text {mes }}$ is the bridge output voltage $(\mathrm{mV})$. $\mathrm{D}_{\mathrm{A}}$ is the displacement of the wire fixation point (Point $\left.A\right)$ which is also the real displacement of the object. If we define the ratio of $D_{A}$ and $D_{B}$ as the compensation factor $C$ on the basis of the model, $C$ can be expressed by $C=D_{A} / D_{B}=1+$ $\left(\mathrm{K}_{2}+\mathrm{K}_{3}\right) / \mathrm{K}_{1}$. $\mathrm{D}_{\mathrm{A}}$ can be calculated by $\mathrm{D}_{\mathrm{A}}=\mathrm{C} \times \mathrm{D}_{\mathrm{B}}=\left(\mathrm{C} \times \mathrm{U}_{\text {mes }}\right) /\left(\mathrm{K}_{0} \times \mathrm{U}_{\mathrm{b}}\right)$.

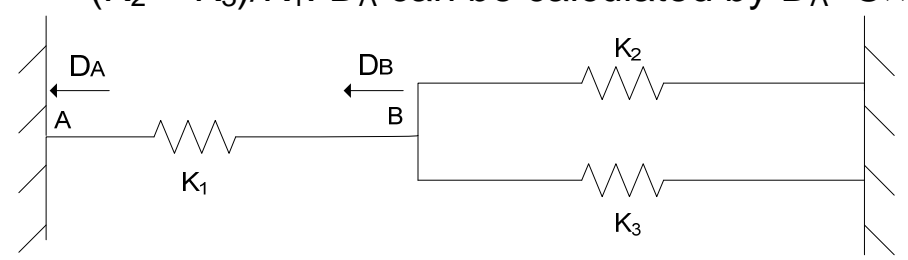

Fig. 5. The simplified mechanical model.

\section{B. Output Properties Study}

In order to study the static transmission properties of the displacement measurement system, we carried out the calibration tests at room temperature (RT). The test mock-up is shown in Fig.6. The cantilever beam was connected to the displacement controller by a copper wire. The resolution of the displacement controller is $5 \mu \mathrm{m}$. During the test, the cantilever CE-10SL (BHZ08646) was connected in 6-wire technique and full bridge. Deformation of measurement mock-up is negligible. The power supply TTI EX354RT was used to provide the exciting voltage. The bridge output voltage was measured by a KEITHLEY 2010 multimeter.

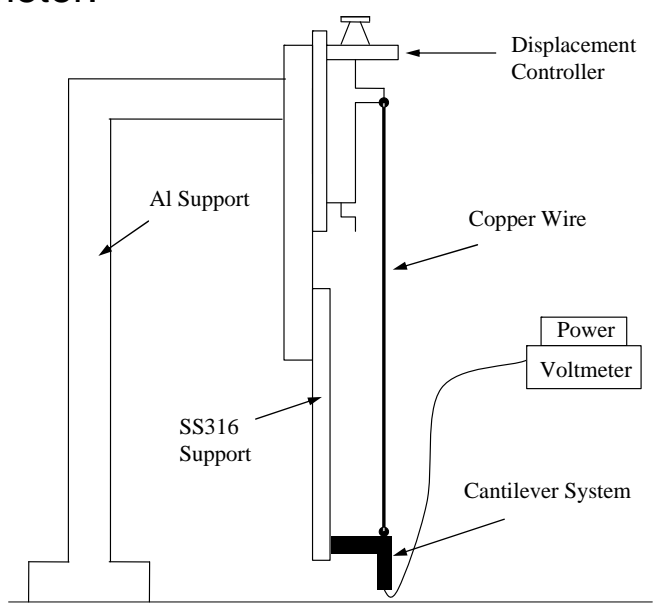

Fig. 6. Experimental setup for RT measurements.

The important output properties of the cantilever system in full range were derived and analysed. One important property is the sensitivity $\left(\mathrm{K}_{0}=\mathrm{U}_{\text {mes }} / \mathrm{U}_{\mathrm{b}} / \mathrm{D}\right)$, which can be derived by linear regression. Based on the test results, we can get the cantilever bridge voltage $U_{\text {mes }}(\mathrm{mV})$ in full range, and the exciting power $U_{\mathrm{b}}$ is already known $(2 \mathrm{~V})$, the transfer function of bridge voltage divided by exciting power $(\mathrm{mV} / \mathrm{V})$ with displacement $\mathrm{D}(\mathrm{mm})$ can be expressed by $U_{\text {mes }} / \cup_{b}=a+b \times D$, where $a$ is the intercept, $b$ the slope of the fitting line (sensitivity $\mathrm{K}_{0}$ ). Fig.7 shows the sensitivity test results at room temperature. The calibrated sensitivity is $-0.3654 \mathrm{mV} / \mathrm{V} / \mathrm{mm}$, which is $6.4 \%$ lower than the sensitivity in transducer specification $(-0.342 \mathrm{mV} / \mathrm{V} / \mathrm{mm})$. 
Another characteristic is the deviation from the linearity, which is the difference between the measured data and the linear fitted data. Fig.8 shows the ratio of deviation and full scale output (FSO) at $293 \mathrm{~K}$, and the maximal value is called non-linearity [4]. In Fig.8, it is clear that the non-linearity is $0.66 \%$ at $\mathrm{RT}$, compared with the transducer specification of $0.4 \%$, it is negligible for application.

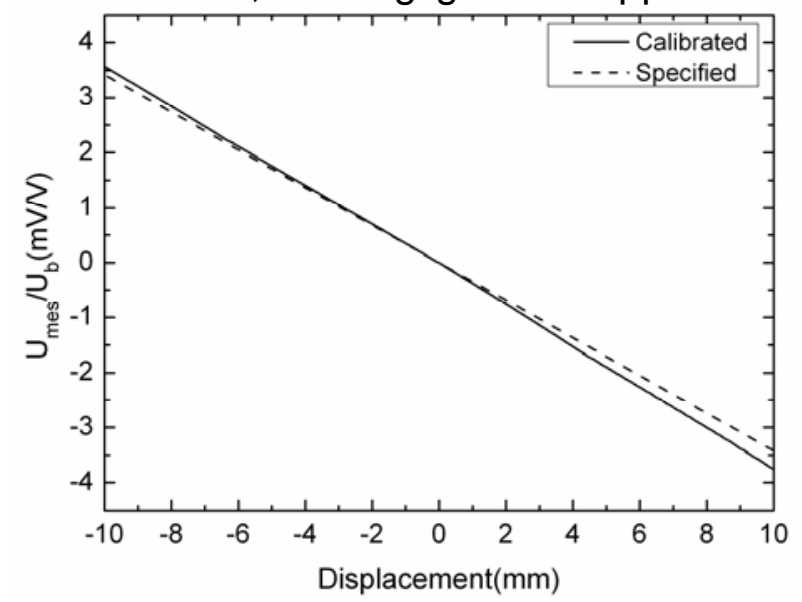

Fig. 7. Bridge voltage divided by power vs. displacement at RT.

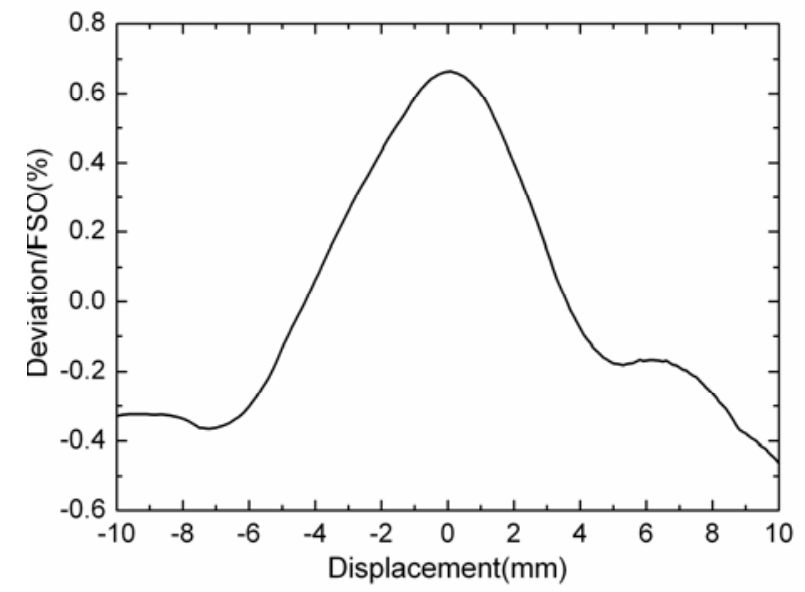

Fig. 8. Deviation divided by FSO vs. displacement at RT.

\section{APPLICATION IN COIL ACCEPTANCE TEST}

In order to verify the quality of the manufactured superconducting coils, all the 70 coils of W7-X were subjected to a test program before installation. The tests were performed in a cryomagnetic test facility at CEA in Saclay [5].

During the cold test phase of the planar coil AAC33, two displacement measurement systems were used to measure the coil deformation. The two cantilever systems were installed on the coil surface through the aluminum frame and thread rod. One system was on the top of the coil, the other was underneath. The copper wire was across the short coil axis. One side of each wire was connected to the corresponding cantilever beam, the other side fixation was located in the middle of the other coil 


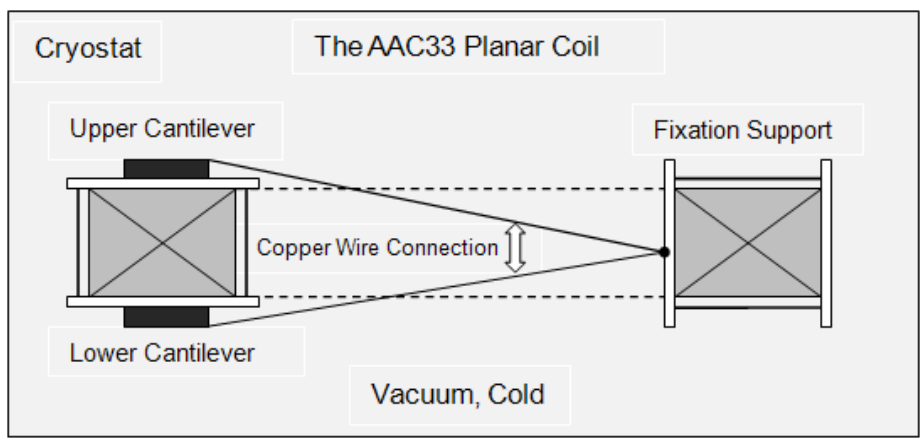

Fig. 9. The installation of the cantilever system on the planar coil AAC33.

side. The overall length of the copper wire was $2455 \mathrm{~mm}$, and the calculated compensation factor $C=1.09$. The cantilever installation on the planar coil AAC33 is presented in Fig.9.

All the cantilever strain signals were recorded by CEA electronics with excitation of $5 \mathrm{~V}$. Due to the facility limitation, the two cantilever systems were connected as half-bridges, and the bridge completion resistors were $350 \Omega$.

\section{A. Displacement Measurement during Cooling Down}

The cool down of the coils is achieved mainly by cooling of the housing. During the cool down phase, the temperature difference between any two points of a coil must not exceed $40 \mathrm{~K}$. The cool down of the planar coil AAC33 from room temperature to liquid helium temperature lasted around 7 days. Fig.10 shows the cool down behavior of the AAC33 winding, which was measured at the winding He-outlet by Cernox sensors. From $300 \mathrm{~K}$ to $125 \mathrm{~K}$, the rate of temperature decrease is about- $1.5 \mathrm{~K} / \mathrm{h}$, while that from $125 \mathrm{~K}$ to $8 \mathrm{~K}$ is about $-2.4 \mathrm{~K} / \mathrm{h}$. Both temperature ramps were coincident with the cooling down specification [6].

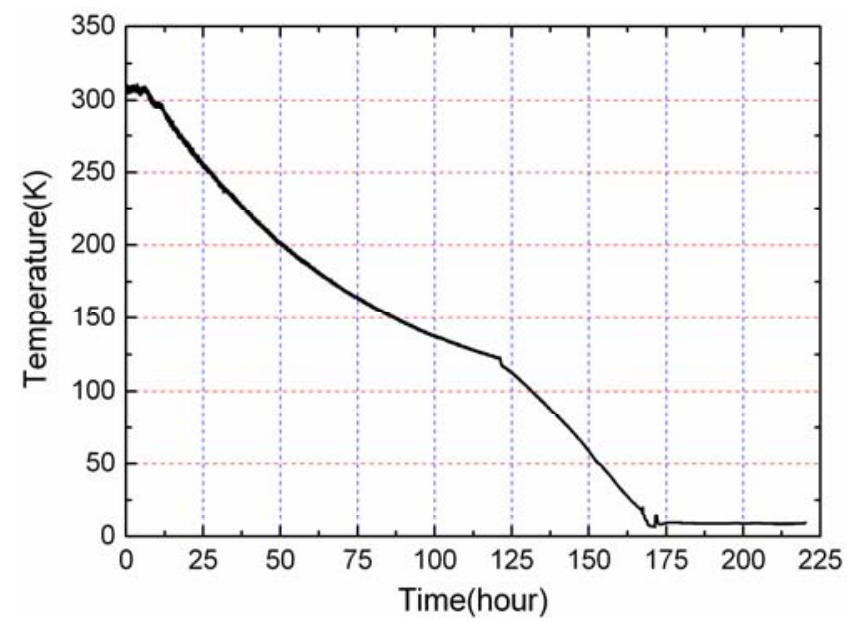

Fig. 10. Cool down process of the AAC33 coil.

During the data analysis, the offset displacement at room temperature was compensated to zero, and the thermal contraction of the connection wire was not taken into account. From the test results, a similar pattern can be found in the two cases of displacement behavior, the peak displacement value is about $1.4 \mathrm{~mm}$ at $225 \mathrm{~K}$. However, at the final 
liquid helium temperature, the two displacements have a deviation up to $0.22 \mathrm{~mm}$. More statistic tests will be carried out to validate whether this deviation is due to the measurement system error.

B. Displacement Measurement during Excitation

The current test of the coil AAC33 lasted two days. In each test day, two current tests with the excitation current up to $16 \mathrm{KA}$ were carried out. Before the excitation process, the coil was ramped up to $1 \mathrm{KA}$ to check the quench detection system.

Due to the similar test procedure on both test days, just the measurement results of the second test day are presented, as shown in Fig.11. It is clear that the two current tests have nearly the same results. After the $1 \mathrm{KA}$ function test, the coil was charged to $10 \mathrm{KA}$ with a ramping rate of $50 \mathrm{~A} / \mathrm{s}$. After the stable operation with $10 \mathrm{KA}$ for about 3 minutes, the coil was further charged with a ramping rate of $20 \mathrm{~A} / \mathrm{s}$ to the maximal current of $16 \mathrm{KA}$. During the plateau at $16 \mathrm{KA}$, the measured displacements of both cantilevers were around $4 \mathrm{~mm}$ (indistinguishable in fig. 11), and the signal noise was up to

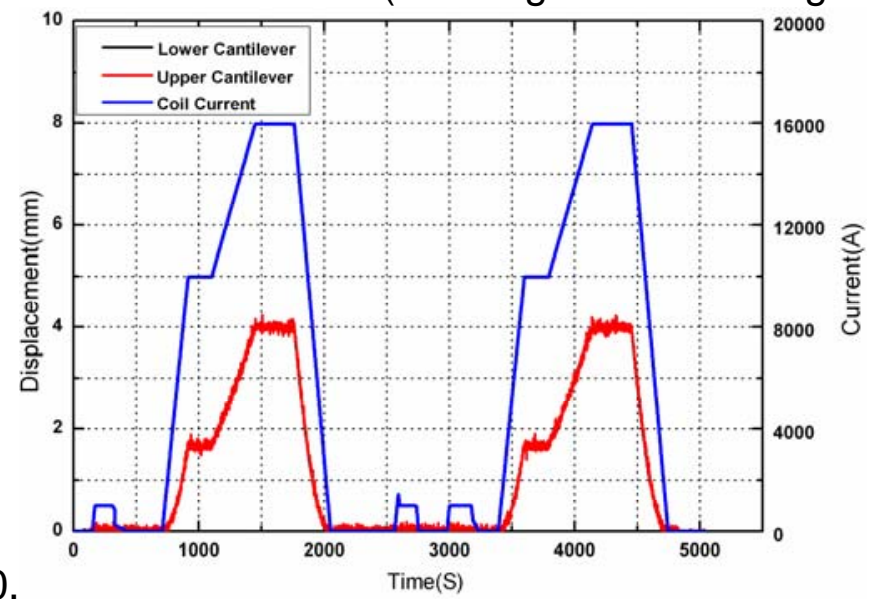

Fig. 11. Profiles of current and cantilever displacements in the second test day.

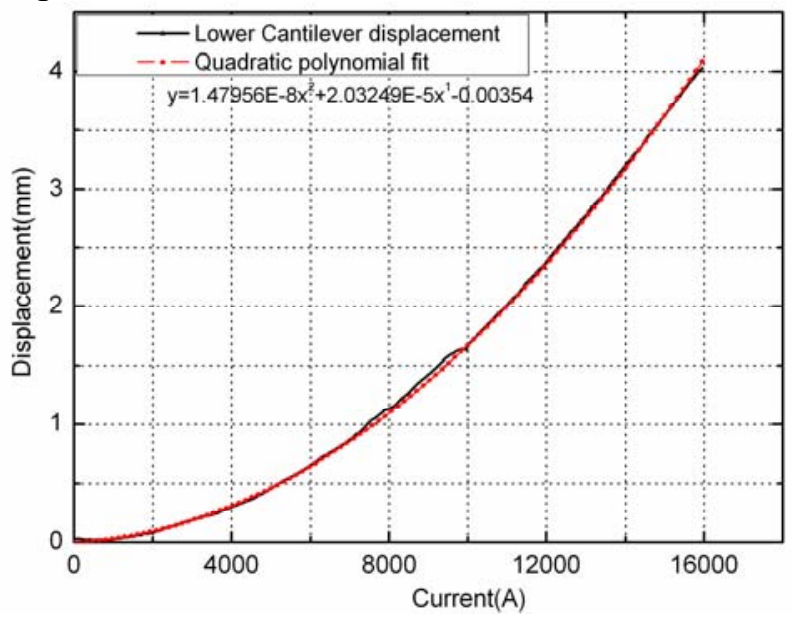

Fig. 12. Lower cantilever displacement variation during the first excitation.

$0.15 \mathrm{~mm}$ equivalent displacement. Compared with the FE predicted value of $3.2 \mathrm{~mm}$, the deviation between measured and calculated results is about $20 \%$ [7]. This deviation is 
acceptable considering the rough FE-model of the coil.

The $16 \mathrm{KA}$ operating current was sustained for about 5 minutes, and then the power supply discharge was initiated with a rate of $-50 \mathrm{~A} / \mathrm{s}$. Electromagnetic forces in the magnet depend on the square of the exciting current which was well reproduced by the cantilever measurements. A typical displacement-current plot is shown in Fig.12.

\section{CONCLUSION}

A commercial cantilever-based displacement measurement system was developed for application in the W7-X superconducting magnet system. The principle and constructive details of the measurement system are presented.

The sensitivity was calibrated at room temperature, and the non-linearity is acceptable for practical application. During the acceptance test phase of the planar coil AAC33, two displacement measurement systems were installed to measure the coil deformation. The first measurement results are reasonable and satisfactory.

\section{ACKNOWLEDGMENT}

The authors would like to sincerely acknowledge the IPP test team for performing the coil acceptance tests at CEA/Saclay. Grateful thanks are given to Dr. F. Schauer at IPP Greifswald for his many valuable suggestions.

\section{REFERENCES}

[1] L. Wegener, "Status of Wendelstein 7-X construction," Fusion Engineering and Design, vol. 84, no. 2-6, pp. 106-112, June 2009.

[2] J. Sapper, W. Gardebrecht, F. Kerl, I. Schoenewolf, "The layout of the WENDELSTEIN 7-X magnet," Fusion Engineering and Design, vol. 58-59, pp. 237-240, November 2001.

[3] Strain Gauge-type Transducers, Tokyo Sokki Kenkyuio Co., Tokyo, Japan, 2007, pp. 53.

[4] M. Milushev, M. Suesser, F. Wuechner, "Investigation of two different types of displacement transducers in the cryogenic environment," Cryogenics, vol. 44, pp. 197-201, March 2004.

[5] H. Viebke, K. Hertel, J. Baldzuhn, G. Croari. L. Genini, B. Renard, L. Vieillard, "Acceptance tests of W7-X coils," Fusion Engineering and Design, vol. 84, no. 7-11, pp. 1925-1927, June 2009.

[6] B. Renard, L. Vieillard, H. Viebke, J.-L. Duchateau, "W7-X superconducting coils cooling at the CEA Saclay cryomagnetic test facility," Fusion Engineering and Design, vol. 84, no. 7-11, pp. 1588-1592, June 2009.

[7] J. P. Kallmeyar, M. Nitz, private communication, Internal IPP Document, March 2009. 